\title{
Search for Dimuon Decays of a Light Scalar Boson in Radiative Transitions $\mathbf{Y} \rightarrow \gamma A^{0}$
}

B. Aubert, ${ }^{1}$ Y. Karyotakis, ${ }^{1}$ J. P. Lees, ${ }^{1}$ V. Poireau, ${ }^{1}$ E. Prencipe,${ }^{1}$ X. Prudent,${ }^{1}$ V. Tisserand, ${ }^{1}$ J. Garra Tico, ${ }^{2}$ E. Grauges ${ }^{2}$ M. Martinelli, ${ }^{3 a, 3 b}$ A. Palano, ${ }^{3 a, 3 b}$ M. Pappagallo, ${ }^{3 a, 3 b}$ G. Eigen, ${ }^{4}$ B. Stugu, ${ }^{4}$ L. Sun,${ }^{4}$ M. Battaglia, ${ }^{5}$ D. N. Brown, ${ }^{5}$

L. T. Kerth, ${ }^{5}$ Yu. G. Kolomensky, ${ }^{5}$ G. Lynch, ${ }^{5}$ I. L. Osipenkov, ${ }^{5}$ E. Petigura, ${ }^{5}$ K. Tackmann, ${ }^{5}$ T. Tanabe, ${ }^{5}$ C. M. Hawkes, ${ }^{6}$

N. Soni, ${ }^{6}$ A. T. Watson, ${ }^{6}$ H. Koch, ${ }^{7}$ T. Schroeder, ${ }^{7}$ D. J. Asgeirsson, ${ }^{8}$ B. G. Fulsom, ${ }^{8}$ C. Hearty, ${ }^{8}$ T. S. Mattison, ${ }^{8}$

J. A. McKenna, ${ }^{8}$ M. Barrett, ${ }^{9}$ A. Khan, ${ }^{9}$ A. Randle-Conde, ${ }^{9}$ V. E. Blinov,${ }^{10}$ A. D. Bukin, ${ }^{10, *}$ A. R. Buzykaev,${ }^{10}$

V.P. Druzhinin, ${ }^{10}$ V. B. Golubev,${ }^{10}$ A. P. Onuchin, ${ }^{10}$ S. I. Serednyakov, ${ }^{10}$ Yu. I. Skovpen, ${ }^{10}$ E. P. Solodov, ${ }^{10}$

K. Yu. Todyshev, ${ }^{10}$ M. Bondioli, ${ }^{11}$ S. Curry, ${ }^{11}$ I. Eschrich, ${ }^{11}$ D. Kirkby, ${ }_{11}^{11}$ A. J. Lankford, ${ }^{11}$ P. Lund, ${ }^{11}$ M. Mandelkern, ${ }^{11}$ E. C. Martin, ${ }^{11}$ D. P. Stoker, ${ }^{11}$ H. Atmacan, ${ }^{12}$ J. W. Gary, ${ }^{12}$ F. Liu, ${ }^{12}$ O. Long, ${ }^{12}$ G. M. Vitug, ${ }^{12}$ Z. Yasin, ${ }^{12}$ V. Sharma,${ }^{13}$

C. Campagnari, ${ }^{14}$ T. M. Hong, ${ }^{14}$ D. Kovalskyi, ${ }^{14}$ M. A. Mazur, ${ }^{14}$ J. D. Richman, ${ }^{14}$ T. W. Beck, ${ }^{15}$ A. M. Eisner ${ }^{15}$

C. A. Heusch, ${ }^{15}$ J. Kroseberg, ${ }^{15}$ W. S. Lockman, ${ }^{15}$ A. J. Martinez, ${ }^{15}$ T. Schalk, ${ }^{15}$ B. A. Schumm, ${ }^{15}$ A. Seiden, ${ }^{15}$ L. Wang, ${ }^{15}$

L. O. Winstrom, ${ }^{15}$ C. H. Cheng, ${ }^{16}$ D. A. Doll, ${ }^{16}$ B. Echenard, ${ }^{16}$ F. Fang, ${ }^{16}$ D. G. Hitlin, ${ }^{16}$ I. Narsky,${ }^{16}$ P. Ongmongkolku, ${ }^{16}$

T. Piatenko, ${ }^{16}$ F. C. Porter, ${ }^{16}$ R. Andreassen,,${ }^{17}$ G. Mancinelli, ${ }^{17}$ B. T. Meadows, ${ }^{17}$ K. Mishra, ${ }^{17}$ M. D. Sokoloff, ${ }^{17}$

P. C. Bloom, ${ }^{18}$ W. T. Ford, ${ }^{18}$ A. Gaz, ${ }^{18}$ J. F. Hirschauer, ${ }^{18}$ M. Nagel,${ }^{18}$ U. Nauenberg, ${ }^{18}$ J. G. Smith, ${ }^{18}$ S. R. Wagner, ${ }^{18}$

R. Ayad, ${ }^{19, \dagger}$ W. H. Toki, ${ }^{19}$ R. J. Wilson, ${ }^{19}$ E. Feltresi, ${ }^{20}$ A. Hauke,${ }^{20}$ H. Jasper, ${ }^{20}$ T. M. Karbach,${ }^{20}$ J. Merkel, ${ }^{20}$ A. Petzold, ${ }^{20}$

B. Spaan, ${ }^{20}$ K. Wacker, ${ }^{20}$ M. J. Kobel, ${ }^{21}$ R. Nogowski, ${ }^{21}$ K. R. Schubert,${ }^{21}$ R. Schwierz, ${ }^{21}$ A. Volk, ${ }^{21}$ D. Bernard ${ }^{22}$

E. Latour, ${ }^{22}$ M. Verderi, ${ }^{22}$ P. J. Clark, ${ }^{23}$ S. Playfer, ${ }^{23}$ J. E. Watson, ${ }^{23}$ M. Andreotti, ${ }^{24 a, 24 b}$ D. Bettoni, ${ }^{24 a}$ C. Bozzi, ${ }^{24 a, 24 b}$

R. Calabrese, ${ }^{24 a, 24 b}$ A. Cecchi, ${ }^{24 a, 24 b}$ G. Cibinetto, ${ }^{24 a, 24 b}$ E. Fioravanti, ${ }^{24 a, 24 b}$ P. Franchini, ${ }^{24 a, 24 b}$ E. Luppi, ${ }^{24 a, 24 b}$

M. Munerato, ${ }^{24 a, 24 b}$ M. Negrini, ${ }^{24 a, 24 a}$ A. Petrella, ${ }^{24 a, 24 b}$ L. Piemontese, ${ }^{24 a}$ V. Santoro, ${ }^{24 a, 24 b}$ R. Baldini-Ferroli, ${ }^{25}$

A. Calcaterra, ${ }^{25}$ R. de Sangro, ${ }^{25}$ G. Finocchiaro, ${ }^{25}$ S. Pacetti, ${ }^{25}$ P. Patteri, ${ }^{25}$ I. M. Peruzzi,${ }^{25}$, M. Piccolo, ${ }^{25}$ M. Rama, ${ }^{25}$

A. Zallo, ${ }^{25}$ R. Contri, ${ }^{26 a, 26 b}$ E. Guido ${ }^{26 a, 26 b}$ M. Lo Vetere ${ }^{26 a, 26 b}$ M. R. Monge ${ }^{26 a, 26 b}$ S. Passaggio, ${ }^{26 a}$ C. Patrignani, ${ }^{26 a, 26 b}$

E. Robutti, ${ }^{26 \mathrm{a}} \mathrm{S}$. Tosi, ${ }^{26 a, 26 \mathrm{~b}} \mathrm{~K}$. S. Chaisanguanthum, ${ }^{27} \mathrm{M}$. Morii, ${ }^{27}$ A. Adametz, ${ }^{28} \mathrm{~J}$. Marks, ${ }^{28} \mathrm{~S}$. Schenk, ${ }^{28} \mathrm{U}$. Uwer, ${ }^{28}$

F. U. Bernlochner, ${ }^{29}$ V. Klose, ${ }^{29}$ H. M. Lacker, ${ }^{29}$ D. J. Bard ${ }^{30}$ P. D. Dauncey, ${ }^{30}$ M. Tibbetts, ${ }^{30}$ P. K. Behera, ${ }^{31}$

M. J. Charles, ${ }^{31}$ U. Mallik, ${ }^{31}$ J. Cochran, ${ }^{32}$ H. B. Crawley, ${ }^{32}$ L. Dong,${ }^{32}$ V. Eyges,${ }^{32}$ W. T. Meyer, ${ }^{32}$ S. Prell, ${ }^{32}$

E. I. Rosenberg, ${ }^{32}$ A. E. Rubin, ${ }^{32}$ Y. Y. Gao, ${ }^{33}$ A. V. Gritsan, ${ }^{33}$ Z. J. Guo, ${ }^{33}$ N. Arnaud, ${ }^{34}$ J. Béquilleux, ${ }^{34}$ A. D' Orazio, ${ }^{34}$ M. Davier,${ }^{34}$ D. Derkach, ${ }^{34}$ J. Firmino da Costa, ${ }^{34}$ G. Grosdidier, ${ }^{34}$ F. Le Diberder, ${ }^{34}$ V. Lepeltier, ${ }^{34}$ A. M. Lutz, ${ }^{34}$ B. Malaescu, ${ }^{34}$ S. Pruvot,${ }^{34}$ P. Roudeau, ${ }^{34}$ M. H. Schune, ${ }^{34}$ J. Serrano, ${ }^{34}$ V. Sordini, ${ }^{34, \S}$ A. Stocchi, ${ }^{34}$ G. Wormser, ${ }^{34}$ D. J. Lange, ${ }^{35}$ D. M. Wright, ${ }^{35}$ I. Bingham, ${ }^{36}$ J. P. Burke, ${ }^{36}$ C. A. Chavez,${ }^{36}$ J. R. Fry, ${ }^{36}$ E. Gabathuler, ${ }^{36}$ R. Gamet, ${ }^{36}$

D. E. Hutchcroft, ${ }^{36}$ D. J. Payne, ${ }^{36}$ C. Touramanis, ${ }^{36}$ A. J. Bevan, ${ }^{37}$ C. K. Clarke,${ }^{37}$ F. Di Lodovico, ${ }^{37}$ R. Sacco, ${ }^{37}$

M. Sigamani, ${ }^{37}$ G. Cowan, ${ }^{38}$ S. Paramesvaran, ${ }^{38}$ A. C. Wren, ${ }^{38}$ D. N. Brown, ${ }^{39}$ C. L. Davis, ${ }^{39}$ A. G. Denig, ${ }^{40}$ M. Fritsch, ${ }^{40}$ W. Gradl ${ }^{40}$ A. Hafner ${ }^{40}$ K. E. Alwyn, ${ }^{41}$ D. Bailey, ${ }^{41}$ R. J. Barlow, ${ }^{41}$ G. Jackson, ${ }^{41}$ G. D. Lafferty, ${ }^{41}$ T. J. West,${ }^{41}$ J. I. Yi ${ }^{41}$ J. Anderson, ${ }^{42}$ C. Chen, ${ }^{42}$ A. Jawahery, ${ }^{42}$ D. A. Roberts, ${ }^{42}$ G. Simi, ${ }^{42}$ J. M. Tuggle,${ }^{42}$ C. Dallapiccola,${ }^{43}$ E. Salvati, ${ }^{43}$

R. Cowan, ${ }^{44}$ D. Dujmic,${ }^{44}$ P. H. Fisher,${ }^{44}$ S. W. Henderson, ${ }^{44}$ G. Sciolla, ${ }^{44}$ M. Spitznagel, ${ }^{44}$ R. K. Yamamoto, ${ }^{44}$ M. Zhao,${ }^{44}$ P. M. Patel, ${ }^{45}$ S. H. Robertson, ${ }^{45}$ M. Schram, ${ }^{45}$ P. Biassoni, ${ }^{46 a, 46 b}$ A. Lazzaro, ${ }^{46 a, 46 b}$ V. Lombardo, ${ }^{46 a}$ F. Palombo, ${ }^{46 a, 46 b}$ S. Stracka, ${ }^{46 a, 46 b}$ J. M. Bauer, ${ }^{47}$ L. Cremaldi,${ }^{47}$ R. Godang, ${ }^{47, \|}$ R. Kroeger, ${ }^{47}$ P. Sonnek, ${ }^{47}$ D. J. Summers, ${ }^{47}$ H. W. Zhao, ${ }^{47}$

M. Simard, ${ }^{48}$ P. Taras ${ }^{48}$ H. Nicholson, ${ }^{49}$ G. De Nardo,${ }^{50 a, 50 b}$ L. Lista, ${ }^{50 a}$ D. Monorchio, ${ }^{50 a, 50 b}$ G. Onorato, ${ }^{50 a, 50 b}$

C. Sciacca, ${ }^{50 a, 50 b}$ G. Raven, ${ }^{51}$ H. L. Snoek,${ }^{51}$ C. P. Jessop, ${ }^{52}$ K. J. Knoepfel, ${ }^{52}$ J. M. LoSecco, ${ }^{52}$ W. F. Wang, ${ }^{52}$ L. A. Corwin, ${ }^{53}$ K. Honscheid, ${ }^{53}$ H. Kagan,${ }^{53}$ R. Kass, ${ }^{53}$ J. P. Morris, ${ }^{53}$ A. M. Rahimi, ${ }^{53}$ J. J. Regensburger, ${ }^{53}$ S. J. Sekula, ${ }^{53}$ Q. K. Wong, ${ }^{53}$ N. L. Blount, ${ }^{54}$ J. Brau, ${ }^{54}$ R. Frey, ${ }^{54}$ O. Igonkina, ${ }^{54}$ J. A. Kolb,${ }^{54}$ M. Lu, ${ }^{54}$ R. Rahmat, ${ }^{54}$ N. B. Sinev, ${ }^{54}$

D. Strom, ${ }^{54}$ J. Strube,${ }^{54}$ E. Torrence, ${ }^{54}$ G. Castelli, ${ }^{55 a, 55 b}$ N. Gagliardi, ${ }^{55 a, 55 b}$ M. Margoni,,${ }^{55 a, 55 b}$ M. Morandin, ${ }^{55 a}$

M. Posocco, ${ }^{55 a}$ M. Rotondo, ${ }^{55 a}$ F. Simonetto, ${ }^{55 a, 55 b}$ R. Stroili, ${ }^{55 a, 55 b}$ C. Voci, ${ }^{55 a, 55 b}$ P. del Amo Sanchez ${ }^{56}$ E. Ben-Haim, ${ }^{56}$ G. R. Bonneaud, ${ }^{56}$ H. Briand, ${ }^{56}$ J. Chauveau, ${ }^{56}$ O. Hamon, ${ }^{56}$ Ph. Leruste, ${ }^{56}$ G. Marchiori, ${ }^{56}$ J. Ocariz,${ }^{56}$ A. Perez ${ }^{56}$

J. Prendki, ${ }^{56}$ S. Sitt, ${ }^{56}$ L. Gladney, ${ }^{57}$ M. Biasini, ${ }^{58 a, 58 b}$ E. Manoni, ${ }^{58 a, 58 b}$ C. Angelini, ${ }^{59 a, 59 b}$ G. Batignani, ${ }^{59 a, 59 b}$

S. Bettarini, ${ }^{59 a, 59 b}$ G. Calderini, ${ }^{59 a, 59 b, \text { Il }}$ M. Carpinelli, ${ }^{59 a, 59 b, * *}$ A. Cervelli, ${ }^{59 a, 59 b}$ F. Forti, ${ }^{59 a, 59 b}$ M. A. Giorgi, ${ }^{59 a, 59 b}$ A. Lusiani, ${ }^{59 a, 59 c}$ M. Morganti, ${ }^{59 a, 59 b}$ N. Neri, ${ }^{59 a, 59 b}$ E. Paoloni, ${ }^{59 a, 59 b}$ G. Rizzo, ${ }^{59 a, 59 b}$ J. J. Walsh,${ }^{59 a}$ D. Lopes Pegna, ${ }^{60}$ C. Lu, ${ }^{60}$ J. Olsen, ${ }^{60}$ A. J. S. Smith,${ }^{60}$ A. V. Telnov,${ }^{60}$ F. Anulli, ${ }^{61 a}$ E. Baracchini, ${ }^{61 a, 61 b}$ G. Cavoto, ${ }^{61 a}$ R. Faccini, ${ }^{61 a, 61 b}$ F. Ferrarotto, ${ }^{61 \mathrm{a}}$ F. Ferroni, ${ }^{61 \mathrm{a}, 61 \mathrm{~b}}$ M. Gaspero, ${ }^{61 \mathrm{a}, 61 \mathrm{~b}}$ P. D. Jackson, ${ }^{61 \mathrm{a}}$ L. Li Gioi, ${ }^{61 \mathrm{a}}$ M. A. Mazzoni, ${ }^{61 \mathrm{a}}$ S. Morganti, ${ }^{61 \mathrm{a}}$ G. Piredda, ${ }^{61 \mathrm{a}}$ F. Renga, ${ }^{61 \mathrm{a}, 61 \mathrm{~b}}$ C. Voena ${ }^{61 \mathrm{a}}$ M. Ebert, ${ }^{62}$ T. Hartmann, ${ }^{62}$ H. Schröder, ${ }^{62}$ R. Waldi, ${ }^{62}$ T. Adye, ${ }^{63}$ B. Franek, ${ }^{63}$ E. O. Olaiya ${ }^{63}$ F. F. Wilson, ${ }^{63}$ S. Emery, ${ }^{64}$ L. Esteve, ${ }^{64}$ G. Hamel de Monchenault, ${ }^{64}$ W. Kozanecki, ${ }^{64}$ G. Vasseur, ${ }^{64}$ 
Ch. Yèche, ${ }^{64}$ M. Zito,${ }^{64}$ M. T. Allen, ${ }^{65}$ D. Aston, ${ }^{65}$ R. Bartoldus, ${ }^{65}$ J. F. Benitez, ${ }^{65}$ R. Cenci, ${ }^{65}$ J. P. Coleman, ${ }^{65}$ M. R. Convery ${ }^{65}$ J. C. Dingfelder ${ }^{65}$ J. Dorfan, ${ }^{65}$ G. P. Dubois-Felsmann, ${ }^{65}$ W. Dunwoodie,${ }^{65}$ R. C. Field ${ }^{65}$ M. Franco Sevilla, ${ }^{65}$ A. M. Gabareen, ${ }^{65}$ M. T. Graham, ${ }^{65}$ P. Grenier, ${ }^{65}$ C. Hast, ${ }^{65}$ W. R. Innes, ${ }^{65}$ J. Kaminski, ${ }^{65}$ M. H. Kelsey, ${ }^{65}$ H. Kim,${ }^{65}$ P. Kim, ${ }^{65}$ M. L. Kocian, ${ }^{65}$ D. W. G. S. Leith ${ }^{65}$ S. Li ${ }^{65}$ B. Lindquist,${ }^{65}$ S. Luitz, ${ }^{65}$ V. Luth, ${ }^{65}$ H. L. Lynch, ${ }^{65}$ D. B. MacFarlane, ${ }^{65}$ H. Marsiske, ${ }^{65}$ R. Messner, ${ }^{65}{ }^{*}$ D. R. Muller ${ }^{65}$ H. Neal,${ }^{65}$ S. Nelson, ${ }^{65}$ C. P. O' Grady, ${ }^{65}$ I. Ofte, ${ }^{65}$ M. Perl, ${ }^{65}$ B. N. Ratcliff, ${ }^{65}$ A. Roodman, ${ }^{65}$ A. A. Salnikov, ${ }^{65}$ R. H. Schindler, ${ }^{65}$ J. Schwiening, ${ }^{65}$ A. Snyder,${ }^{65}$ D. Su, ${ }^{65}$ M. K. Sullivan, ${ }^{65}$ K. Suzuki,${ }^{65}$ S. K. Swain, ${ }^{65}$ J. M. Thompson, ${ }^{65}$ J. Va'vra, ${ }^{65}$ A. P. Wagner, ${ }^{65}$ M. Weaver ${ }^{65}$ C. A. West, ${ }^{65}$ W. J. Wisniewski, ${ }^{65}$ M. Wittgen,${ }^{65}$ D. H. Wright, ${ }^{65}$ H. W. Wulsin, ${ }^{65}$ A. K. Yarritu ${ }^{65}$ C. C. Young, ${ }^{65}$ V. Ziegler, ${ }^{65}$ X. R. Chen,${ }^{66}$ H. Liu, ${ }^{66}$ W. Park ${ }^{66}$ M. V. Purohit ${ }^{66}$ R. M. White, ${ }^{66}$ J. R. Wilson, ${ }^{66}$ P. R. Burchat, ${ }^{67}$ A. J. Edwards, ${ }^{67}$ T. S. Miyashita,${ }^{67}$ S. Ahmed, ${ }^{68}$ M. S. Alam, ${ }^{68}$ J. A. Ernst, ${ }^{68}$ B. Pan,${ }^{68}$ M. A. Saeed, ${ }^{68}$ S. B. Zain, ${ }^{68}$ A. Soffer, ${ }^{69}$ S. M. Spanier ${ }^{70}$ B. J. Wogsland,${ }^{70}$ R. Eckmann, ${ }^{71}$ J. L. Ritchie, ${ }^{71}$ A. M. Ruland, ${ }^{71}$ C. J. Schilling, ${ }^{71}$ R. F. Schwitters, ${ }^{71}$ B. C. Wray, ${ }^{71}$ B. W. Drummond,${ }^{72}$ J. M. Izen, ${ }^{72}$ X. C. Lou, ${ }^{72}$ F. Bianchi, ${ }^{73 a, 73 b}$ D. Gamba, ${ }^{73 a, 73 b}$ M. Pelliccioni, ${ }^{73 a, 73 b}$ M. Bomben, ${ }^{74 a, 74 b}$ L. Bosisio, ${ }^{74 a, 74 b}$ C. Cartaro, ${ }^{74 a, 74 b}$ G. Della Ricca, ${ }^{74 a, 74 b}$ L. Lanceri, ${ }^{74 a, 74 b}$ L. Vitale ${ }^{74 a, 74 b}$ V. Azzolini, ${ }^{75}$ N. Lopez-March,${ }^{75}$ F. Martinez-Vidal,${ }^{75}$ D. A. Milanes, ${ }^{75}$ A. Oyanguren, ${ }^{75}$ J. Albert, ${ }^{76}$ Sw. Banerjee, ${ }^{76}$ B. Bhuyan, ${ }^{76}$ H. H. F. Choi, ${ }^{76}$ K. Hamano, ${ }^{76}$ G. J. King, ${ }^{76}$ R. Kowalewski, ${ }^{76}$ M. J. Lewczuk, ${ }^{76}$ I. M. Nugent, ${ }^{76}$ J. M. Roney, ${ }^{76}$ R. J. Sobie, ${ }^{76}$ T. J. Gershon, ${ }^{77}$ P. F. Harrison, ${ }^{77}$ J. Ilic, ${ }^{77}$ T. E. Latham, ${ }^{77}$ G. B. Mohanty, ${ }^{77}$ E. M. T. Puccio, ${ }^{77}$ H. R. Band, ${ }^{78}$ X. Chen, ${ }^{78}$ S. Dasu, ${ }^{78}$ K. T. Flood,${ }^{78}$ Y. Pan, ${ }^{78}$ R. Prepost,${ }^{78}$ C. O. Vuosalo, ${ }^{78}$ and S. L. $\mathrm{Wu}^{78}$

(BABAR Collaboration)

${ }^{1}$ Laboratoire d'Annecy-le-Vieux de Physique des Particules (LAPP), Université de Savoie, CNRS/IN2P3, F-74941 Annecy-Le-Vieux, France

${ }^{2}$ Universitat de Barcelona, Facultat de Fisica, Departament ECM, E-08028 Barcelona, Spain

${ }^{3 a}$ INFN Sezione di Bari, I-70126 Bari, Italy

${ }^{3 \mathrm{~b}}$ Dipartimento di Fisica, Università di Bari, I-70126 Bari, Italy

${ }^{4}$ University of Bergen, Institute of Physics, N-5007 Bergen, Norway

${ }^{5}$ Lawrence Berkeley National Laboratory and University of California, Berkeley, California 94720, USA

${ }^{6}$ University of Birmingham, Birmingham, B15 2TT, United Kingdom

${ }^{7}$ Ruhr Universität Bochum, Institut für Experimentalphysik 1, D-44780 Bochum, Germany

${ }^{8}$ University of British Columbia, Vancouver, British Columbia, Canada V6T 1Z1

${ }^{9}$ Brunel University, Uxbridge, Middlesex UB8 3PH, United Kingdom

${ }^{10}$ Budker Institute of Nuclear Physics, Novosibirsk 630090, Russia

${ }^{11}$ University of California at Irvine, Irvine, California 92697, USA

${ }^{12}$ University of California at Riverside, Riverside, California 92521, USA

${ }^{13}$ University of California at San Diego, La Jolla, California 92093, USA

${ }^{14}$ University of California at Santa Barbara, Santa Barbara, California 93106, USA

${ }^{15}$ University of California at Santa Cruz, Institute for Particle Physics, Santa Cruz, California 95064, USA

${ }^{16}$ California Institute of Technology, Pasadena, California 91125, USA

${ }^{17}$ University of Cincinnati, Cincinnati, Ohio 45221, USA

${ }^{18}$ University of Colorado, Boulder, Colorado 80309, USA

${ }^{19}$ Colorado State University, Fort Collins, Colorado 80523, USA

${ }^{20}$ Technische Universität Dortmund, Fakultät Physik, D-44221 Dortmund, Germany

${ }^{21}$ Technische Universität Dresden, Institut für Kern- und Teilchenphysik, D-01062 Dresden, Germany

${ }^{22}$ Laboratoire Leprince-Ringuet, CNRS/IN2P3, Ecole Polytechnique, F-91128 Palaiseau, France

${ }^{23}$ University of Edinburgh, Edinburgh EH9 3JZ, United Kingdom

${ }^{24 a}$ INFN Sezione di Ferrara, I-44100 Ferrara, Italy

${ }^{24 \mathrm{~b}}$ Dipartimento di Fisica, Università di Ferrara, I-44100 Ferrara, Italy

${ }^{25}$ INFN Laboratori Nazionali di Frascati, I-00044 Frascati, Italy

${ }^{26 a}$ INFN Sezione di Genova, I-16146 Genova, Italy

${ }^{26 \mathrm{~b}}$ Dipartimento di Fisica, Università di Genova, I-16146 Genova, Italy

${ }^{27}$ Harvard University, Cambridge, Massachusetts 02138, USA

${ }^{28}$ Universität Heidelberg, Physikalisches Institut, Philosophenweg 12, D-69120 Heidelberg, Germany

${ }^{29}$ Humboldt-Universität zu Berlin, Institut für Physik, Newtonstraße 15, D-12489 Berlin, Germany

${ }^{30}$ Imperial College London, London, SW7 2AZ, United Kingdom

${ }^{31}$ University of Iowa, Iowa City, Iowa 52242, USA

${ }^{32}$ Iowa State University, Ames, Iowa 50011-3160, USA 


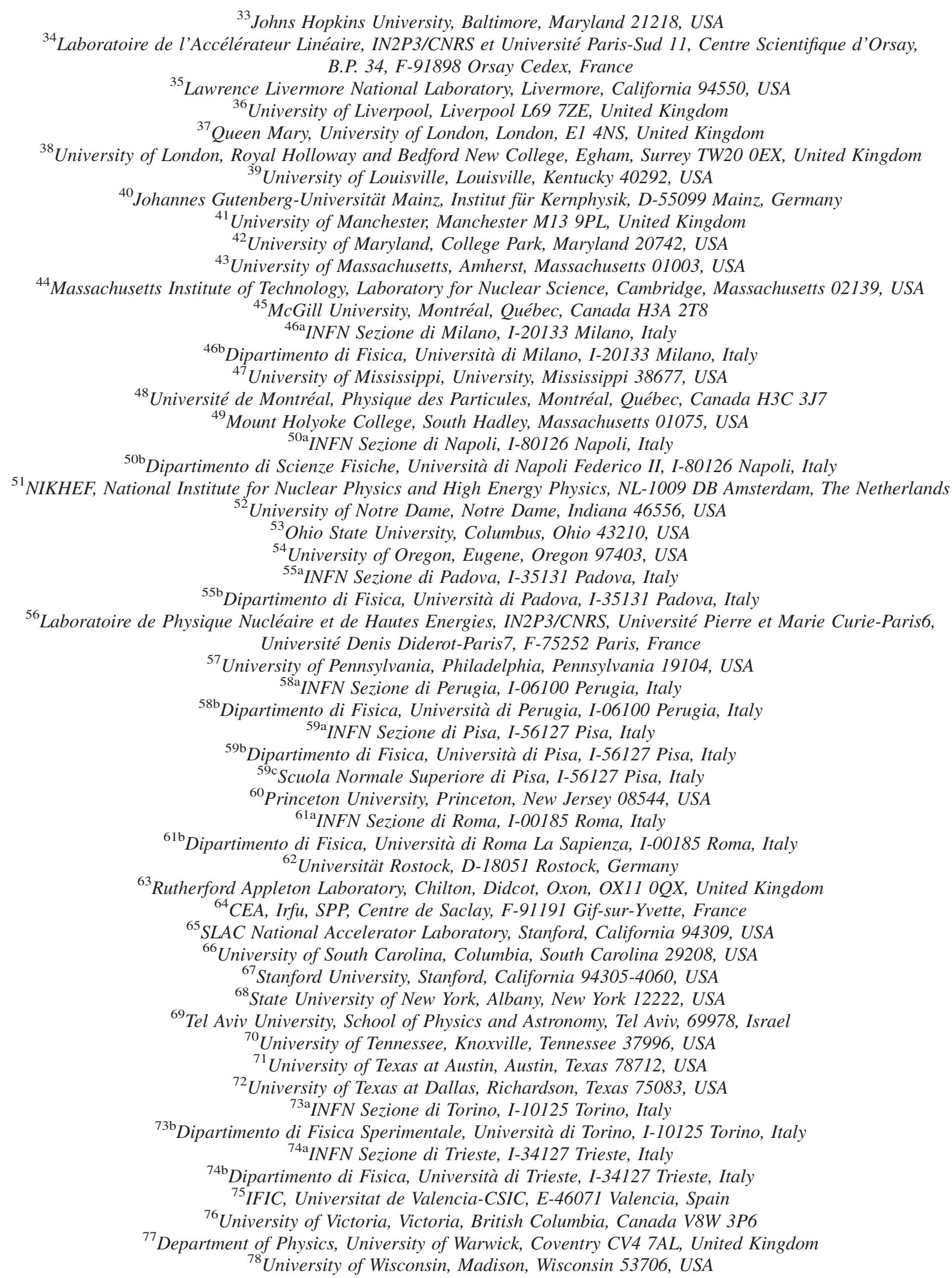

(Received 27 May 2009; published 18 August 2009)

We search for evidence of a light scalar boson in the radiative decays of the $Y(2 S)$ and $Y(3 S)$ resonances: $Y(2 S, 3 S) \rightarrow \gamma A^{0}, A^{0} \rightarrow \mu^{+} \mu^{-}$. Such a particle appears in extensions of the standard model, 
where a light $C P$-odd Higgs boson naturally couples strongly to $b$ quarks. We find no evidence for such processes in the mass range $0.212 \leq m_{A^{0}} \leq 9.3 \mathrm{GeV}$ in the samples of $99 \times 10^{6} Y(2 S)$ and $122 \times 10^{6}$ $Y(3 S)$ decays collected by the BABAR detector at the SLAC PEP-II $B$ factory and set stringent upper limits on the effective coupling of the $b$ quark to the $A^{0}$. We also limit the dimuon branching fraction of the $\eta_{b}$ meson: $\mathcal{B}\left(\eta_{b} \rightarrow \mu^{+} \mu^{-}\right)<0.9 \%$ at $90 \%$ confidence level.

DOI: 10.1103/PhysRevLett.103.081803

The concept of mass is one of the most intuitive ideas in physics since it is present in everyday human experience. Yet the fundamental nature of mass remains one of the great mysteries of science. The Higgs mechanism is a theoretically appealing way to account for the different masses of elementary particles [1]. It implies the existence of at least one new scalar particle, the Higgs boson, which is the only standard model (SM) [2] particle yet to be observed. The SM Higgs boson mass is constrained to be of $O(100-200 \mathrm{GeV})$ by direct searches [3] and by precision electroweak measurements [4].

A number of theoretical models extend the Higgs sector to include additional Higgs fields, some of them naturally light [5]. Similar light scalar states, e.g., axions, appear in models motivated by astrophysical observations and are typically assumed to have Higgs-like couplings [6]. Direct searches typically constrain the mass of such a light particle $A^{0}$ to be below $2 m_{b}$ [7], making it accessible to radiative decays of $Y$ resonances [8]. Model predictions for the branching fraction (BF) of $Y \rightarrow \gamma A^{0}$ decays range from $10^{-6}[6,9]$ to as high as $10^{-4}$ [9]. Empirical motivation for a low-mass Higgs search comes from the HyperCP experiment [10], which observed three anomalous events in the $\Sigma^{+} \rightarrow p \mu^{+} \mu^{-}$final state. These events have been interpreted as production of a scalar boson with the mass of 214.3 MeV decaying into a pair of muons [11,12]. The large data sets available at $B A B A R$ allow us to place stringent constraints on such models.

If a light scalar $A^{0}$ exists, the pattern of its decays depends on its mass. Assuming no invisible (neutralino) decays [13], for low masses $m_{A^{0}}<2 m_{\tau}$ the $\mathrm{BF} \mathcal{B}_{\mu \mu} \equiv$ $\mathcal{B}\left(A^{0} \rightarrow \mu^{+} \mu^{-}\right)$should be sizable. Significantly above the $\tau$ threshold, $A^{0} \rightarrow \tau^{+} \tau^{-}$would dominate [14,15], and hadronic decays might also be significant.

This Letter describes a search for a resonance in the dimuon invariant mass distribution for the fully reconstructed final state $Y(2 S, 3 S) \rightarrow \gamma A^{0}, A^{0} \rightarrow \mu^{+} \mu^{-}$. We assume that the decay width of the $A^{0}$ resonance is negligibly small compared with the experimental resolution, as expected $[6,16]$ for $m_{A^{0}}$ sufficiently far from the mass of the $\eta_{b}$ [17]. We further assume that the resonance is a scalar (or pseudoscalar) particle. While the significance of any observation would not depend on this assumption, the signal efficiency and, therefore, the BFs are computed for a spin-0 particle. In addition, following the recent discovery of the $\eta_{b}$ meson [17], we look for the leptonic decay of the $\eta_{b}$ through $Y(2 S, 3 S) \rightarrow \gamma \eta_{b}, \quad \eta_{b} \rightarrow \mu^{+} \mu^{-}$. We use
PACS numbers: 14.80.Cp, 13.20.Gd, 14.40.Gx, 14.80.Mz

$\Gamma\left(\eta_{b}\right)=10 \pm 5 \mathrm{MeV}$, the range expected in most theoretical models and consistent with the BABAR results [17].

We search for two-body transitions $Y(2 S, 3 S) \rightarrow \gamma A^{0}$, followed by decay $A^{0} \rightarrow \mu^{+} \mu^{-}$in samples of $(98.6 \pm$ $0.9) \times 10^{6} Y(2 S)$ and $(121.8 \pm 1.2) \times 10^{6} Y(3 S)$ decays collected with the BABAR detector at the PEP-II asymmetric-energy $e^{+} e^{-}$collider at the SLAC National Accelerator Laboratory. We use a sample of $79 \mathrm{fb}^{-1}$ accumulated on the $Y(4 S)$ resonance [ $Y(4 S)$ sample] for studies of the continuum backgrounds. Since the $Y(4 S)$ is 3 orders of magnitude broader than the $Y(2 S)$ and $Y(3 S)$, the $\mathrm{BF} \mathcal{B}\left(Y(4 S) \rightarrow \gamma A^{0}\right)$ is expected to be negligible. For characterization of the background events and selection optimization, we also use a sample of $1.4 \mathrm{fb}^{-1}\left(2.4 \mathrm{fb}^{-1}\right)$ collected $30 \mathrm{MeV}$ below the $Y(2 S)[Y(3 S)]$ resonance (offresonance samples). The BABAR detector is described in detail elsewhere $[18,19]$.

We select events with exactly two oppositely charged tracks and a single energetic photon with a center-of-mass (c.m.) energy $E_{\gamma}^{*} \geq 0.2 \mathrm{GeV}$, while allowing additional photons with c.m. energies below $0.2 \mathrm{GeV}$ to be present in the event. We assign a muon mass hypothesis to the two tracks (henceforth referred to as muon candidates) and require that at least one is positively identified as a muon [19]. We require that the muon candidates form a geometric vertex with $\chi_{\mathrm{vtx}}^{2}<20$ for 1 degree of freedom and displaced transversely by at most $2 \mathrm{~cm}$ [20] from the nominal location of the $e^{+} e^{-}$interaction region. We perform a kinematic fit to the $Y$ candidate formed from the two muon candidates and the energetic photon. The c.m. energy of the $Y$ candidate is constrained, within the beam energy spread, to the total beam energy $\sqrt{s}$, and the decay vertex of the $Y$ is constrained to the beam interaction region. We select events with $-0.2<\sqrt{s}-m(\mathcal{Y})<$ $0.6 \mathrm{GeV}$ and place a requirement on the kinematic fit $\chi_{Y}^{2}<$ 30 (for 6 degrees of freedom). We further require that the momenta of the dimuon candidate $A^{0}$ and the photon are back-to-back in the c.m. frame to within $0.07 \mathrm{rad}$ and that the cosine of the angle between the muon direction and $A^{0}$ direction in the center of mass of the $A^{0}$ is less than 0.92 . The selection criteria are chosen to maximize $\varepsilon / \sqrt{B}$, where $\varepsilon$ is the average selection efficiency for a broad $m_{A^{0}}$ range and $B$ is the background yield in the off-resonance sample.

The criteria above select $387546 \quad Y(2 S)$ and $724551 Y(3 S)$ events [mass spectra for $Y(2 S)$ and $Y(3 S)$ data sets are shown in Fig. 1 in [21]]. The backgrounds are dominated by two types of QED processes: "continuum" 
$e^{+} e^{-} \rightarrow \gamma \mu^{+} \mu^{-}$and the initial-state radiation (ISR) production of $\rho^{0}, \phi, J / \psi, \psi(2 S)$, and $Y(1 S)$ vector mesons. In order to suppress contributions from the ISR-produced $\rho^{0} \rightarrow \pi^{+} \pi^{-}$final state in which a pion is misidentified as a muon (probability $\sim 3 \% /$ pion), we require that both tracks are positively identified as muons when we search for $A^{0}$ candidates in the range $0.5 \leq m_{A^{0}}<1.05 \mathrm{GeV}$. Finally, when selecting candidate events in the $\eta_{b}$ region with dimuon invariant mass $m_{\mu \mu} \sim 9.39 \mathrm{GeV}$ in the $Y(2 S)$ $[Y(3 S)]$ data set, we suppress the decay chain $Y(2 S) \rightarrow$ $\gamma_{2} \chi_{b}(1 P), \quad \chi_{b}(1 P) \rightarrow \gamma_{1} Y(1 S) \quad\left[Y(3 S) \rightarrow \gamma_{2} \chi_{b}(2 P)\right.$, $\left.\chi_{b}(2 P) \rightarrow \gamma_{1} Y(1 S)\right]$ by requiring that no secondary photon $\gamma_{2}$ above a c.m. energy of $E_{2}^{*}=0.1 \mathrm{GeV}(0.08 \mathrm{GeV})$ is present in the event.

We use signal Monte Carlo (MC) samples [22,23] $Y(2 S) \rightarrow \gamma A^{0}$ and $Y(3 S) \rightarrow \gamma A^{0}$ generated at 20 values of $m_{A^{0}}$ over a broad range $0.212 \leq m_{A^{0}} \leq 9.5 \mathrm{GeV}$ to measure the selection efficiency for the signal events. The efficiency varies between $24 \%$ and $55 \%$, depending on $m_{A^{0}}$.

We extract the yield of signal events as a function of $m_{A^{0}}$ in the interval $0.212 \leq m_{A^{0}} \leq 9.3 \mathrm{GeV}$ by performing a series of unbinned extended maximum likelihood fits to the distribution of the reduced mass $m_{R} \equiv \sqrt{m_{\mu \mu}^{2}-4 m_{\mu}^{2}}$. The likelihood function contains contributions from the signal, continuum background, and, where appropriate, peaking backgrounds, as described below. For $0.212 \leq m_{A^{0}}<$ $0.5 \mathrm{GeV}$, we fit over a fixed interval $0.01<m_{R}<$ $0.55 \mathrm{GeV}$; near the $J / \psi$ resonance, we fit over the interval $2.7<m_{R}<3.5 \mathrm{GeV}$; and near the $\psi(2 S)$ resonance, we fit over the range $3.35<m_{R}<4.1 \mathrm{GeV}$. Elsewhere, we use sliding intervals $\mu-0.2<m_{R}<\mu+0.1 \mathrm{GeV}$, where $\mu$ is the mean of the signal distribution of $m_{R}$. We search for $A^{0}$ in fine mass steps $\Delta m_{A^{0}}=2-5 \mathrm{MeV}$. We sample a total of $1951 m_{A^{0}}$ values. For each $m_{A^{0}}$ value, we determine the BF products $\mathcal{B}_{n S} \equiv \mathcal{B}\left(Y(n S) \rightarrow \gamma A^{0}\right) \times \mathcal{B}_{\mu \mu}$, where $n=$ 2,3 . Both the fitting procedure and the event selection were developed and tested using MC and $Y(4 S)$ samples prior to their application to the $Y(2 S)$ and $Y(3 S)$ data sets.

The signal probability density function (PDF) is described by a sum of two Crystal Ball functions [24] with tail parameters on either side of the maximum. The signal PDFs are centered around the expected values of $m_{R}$ and have a typical resolution of 2-10 $\mathrm{MeV}$, which increases monotonically with $m_{A^{0}}$. We determine the PDF as a function of $m_{A^{0}}$ using the signal $\mathrm{MC}$ samples, and we interpolate PDF parameters and signal efficiency values linearly between the simulated points. We determine the uncertainty in the PDF parameters by comparing the distributions of the simulated and reconstructed $e^{+} e^{-} \rightarrow$ $\gamma_{\mathrm{ISR}} J / \psi, J / \psi \rightarrow \mu^{+} \mu^{-}$events.

We describe the continuum background below $m_{R}<$ $0.23 \mathrm{GeV}$ with a threshold function $f_{\mathrm{bkg}}\left(m_{R}\right) \propto$ $\tanh \left(\sum_{\ell=1}^{3} p_{\ell} m_{R}^{\ell}\right)$. The parameters $p_{\ell}$ are fixed to the values determined from the fits to the $e^{+} e^{-} \rightarrow \gamma \mu^{+} \mu^{-} \mathrm{MC}$ sample [25] and agree, within statistics, with those determined by fitting the $Y(2 S), Y(3 S)$, and $Y(4 S)$ samples with the signal contribution set to zero. Elsewhere the background is well described in each limited $m_{R}$ range by a first-order $\left(m_{R}<9.3 \mathrm{GeV}\right)$ or a second-order $\left(m_{R}>9.3 \mathrm{GeV}\right)$ polynomial with coefficients determined by the fit.

Events due to known resonances $\phi, J / \psi, \psi(2 S)$, and $\Upsilon(1 S)$ are present in our sample in specific $m_{R}$ intervals and constitute peaking backgrounds. We include these contributions in the fit where appropriate and describe the shape of the resonances using the same functional form as for the signal, a sum of two Crystal Ball functions, with parameters determined from fits to the combined $Y(2 S)$ and $Y(3 S)$ data set. The contribution to the event yield from $\phi \rightarrow K^{+} K^{-}$, in which one of the kaons is misidentified as a muon, is fixed to $111 \pm 24[Y(2 S)]$ and $198 \pm 42[Y(3 S)]$. We determine this contribution from the event yield of $e^{+} e^{-} \rightarrow \gamma \phi, \phi \rightarrow K^{+} K^{-}$in a sample where both kaons are positively identified, corrected for the measured misidentification rate of kaons as muons. We do not search for $A^{0}$ candidates in the immediate vicinity of $J / \psi$ and $\psi(2 S)$, excluding regions of $\pm 40 \mathrm{MeV}$ around $J / \psi(\approx \pm 5 \sigma)$ and $\pm 25 \mathrm{MeV}$ $(\approx \pm 3 \sigma)$ around $\psi(2 S)$.

We compare the overall selection efficiency between the data and the MC simulation by measuring the absolute cross section $d \sigma / d m_{R}$ for the radiative QED process $e^{+} e^{-} \rightarrow \gamma \mu^{+} \mu^{-}$over the broad kinematic range $0<$ $m_{R} \leq 9.6 \mathrm{GeV}$, using the off-resonance sample. We use the ratio of measured to expected [25] cross sections to correct the signal selection efficiency as a function of $m_{A^{0}}$. This correction ranges between $4 \%$ and $10 \%$, with a systematic uncertainty of $5 \%$. This uncertainty accounts for effects of selection, reconstruction (for both charged tracks and the photon), and trigger efficiencies.

We determine the uncertainty in the signal and peaking background PDFs by comparing the distributions of $\approx 4000$ data and $\mathrm{MC} e^{+} e^{-} \rightarrow \gamma_{\mathrm{ISR}} J / \psi, J / \psi \rightarrow \mu^{+} \mu^{-}$ events. We correct for the observed difference in the width of the $m_{R}$ distribution (5.3 MeV in MC simulations versus 6.6 MeV in the data) and use half of the correction to estimate the systematic uncertainty on the signal yield. This is the dominant systematic uncertainty on the signal yield for $m_{A^{0}}>0.4 \mathrm{GeV}$. We estimate that the uncertainties in the tail parameters of the Crystal Ball PDF contribute less than $1 \%$ to the uncertainty in signal yield based on the observed variations in the $J / \psi$ yield. The systematic uncertainties due to the fixed continuum background PDF for $m_{R}<0.23$ and the fixed contribution from $e^{+} e^{-} \rightarrow$ $\gamma \phi$ do not exceed $\sigma_{\text {bkg }}\left(\mathcal{B}_{n S}\right)=0.2 \times 10^{-6}$. These are the largest systematic contributions for $0.212 \leq m_{A^{0}}<$ $0.4 \mathrm{GeV}$.

We test for possible bias in the fitted value of the signal yield with a large ensemble of pseudoexperiments. The 
bias is consistent with zero for all values of $m_{A^{0}}$, and we assign a BF uncertainty of $\sigma_{\text {bias }}\left(\mathcal{B}_{n S}\right)=0.05 \times 10^{-6}$ at all values of $m_{A^{0}}$ to cover the statistical variations in the results of the test.

To estimate the significance of any positive fluctuation, we compute the likelihood ratio variable $\mathcal{S}\left(m_{A^{0}}\right)=$ $\operatorname{sgn}\left(N_{\text {sig }}\right) \sqrt{2 \log \left(L_{\max } / L_{0}\right)}$, where $L_{\max }$ is the maximum likelihood value for a fit with a free signal yield centered at $m_{A^{0}}, N_{\text {sig }}$ is that fitted signal yield, and $L_{0}$ is the value of the likelihood for the signal yield fixed at zero. Under the null hypothesis $\mathcal{S}$ is expected to be normal-distributed with $\mu=0$ and $\sigma=1$ (Fig. 1). Including systematics, the largest $\mathcal{S}$ values are $3.1[Y(2 S)]$ and $2.8[Y(3 S)]$, consistent with a null-hypothesis distribution for $1951 m_{A^{0}}$ points.

Since we do not observe a significant excess of events above the background in the range $0.212<m_{A^{0}} \leq$ $9.3 \mathrm{GeV}$, we set upper limits on $\mathcal{B}_{2 S}$ and $\mathcal{B}_{3 S}$. We add statistical and systematic uncertainties in quadrature. The 90\% confidence level (C.L.) Bayesian upper limits, computed with a uniform prior and assuming a Gaussian likelihood function, are shown in Fig. 2 as a function of mass $m_{A^{0}}$. The limits vary from $0.26 \times 10^{-6}$ to $8.3 \times 10^{-6}$ $\left(\mathcal{B}_{2 S}\right)$ and from $0.27 \times 10^{-6}$ to $5.5 \times 10^{-6}\left(\mathcal{B}_{3 S}\right)$.

The BFs $\mathcal{B}\left(Y(n S) \rightarrow \gamma A^{0}\right)$ are related to the effective coupling $f_{Y}$ of the bound $b$ quark to the $A^{0}$ through $[8,12,26]$

$$
\frac{\mathcal{B}\left(Y(n S) \rightarrow \gamma A^{0}\right)}{\mathcal{B}\left(Y(n S) \rightarrow l^{+} l^{-}\right)}=\frac{f_{Y}^{2}}{2 \pi \alpha}\left(1-\frac{m_{A^{0}}^{2}}{m_{Y(n S)}^{2}}\right)
$$

where $l \equiv e$ or $\mu$ and $\alpha$ is the fine structure constant. The effective coupling $f_{Y}$ includes the Yukawa coupling of the $b$ quark and the $m_{A^{0}}$-dependent QCD and relativistic corrections to $\mathcal{B}_{n S}$ [26] and the leptonic width of $Y(n S)$ [27]. To first order in $\alpha_{S}$, the corrections range from 0 to $30 \%$ [26] but have comparable uncertainties [28]. The ratio of corrections for $Y(2 S)$ and $Y(3 S)$ is within $4 \%$ of unity [26] in the relevant range of $m_{A^{0}}$. We do not attempt to factorize these contributions but instead compute the experimentally accessible quantity $f_{Y}^{2} \mathcal{B}_{\mu \mu}$ and average $\mathcal{Y}(2 S)$ and $\mathcal{Y}(3 S)$

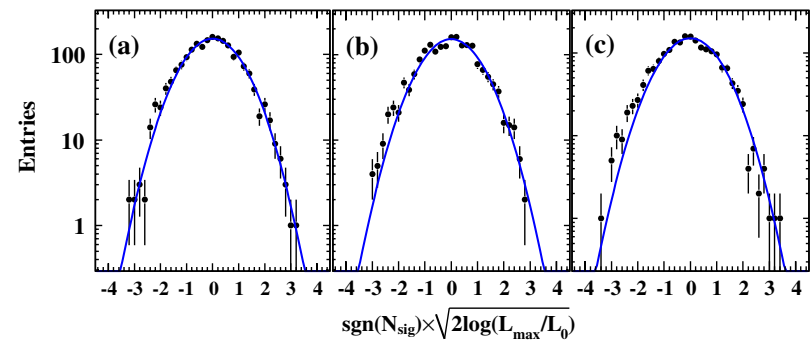

FIG. 1 (color online). Distribution of the log-likelihood variable $\mathcal{S}$ with both statistical and systematic uncertainties included for a (a) $Y(2 S)$ fit, (b) $Y(3 S)$ fit, and (c) combination of $Y(2 S)$ and $Y(3 S)$ data. There are no points outside of the displayed region of $\mathcal{S}$. The solid curve is the standard normal distribution. results, taking into account both correlated and uncorrelated uncertainties. The combined upper limits are shown as a function of $m_{A^{0}}$ in Fig. 2(c) (plots with expanded mass scales in three ranges of $m_{A^{0}}$ are available in Figs. 2-4 in [21]) and span the range $(0.44-44) \times 10^{-6}$, at $90 \%$ C.L. The combined likelihood variable $\langle\mathcal{S}\rangle=\left(w_{2 S} \mathcal{S}_{2 S}+\right.$ $\left.w_{3 S} \mathcal{S}_{3 S}\right) / \sqrt{w_{2 S}^{2}+w_{3 S}^{2}}$ is shown in Fig. 1(c), where $w_{n S}$ is the statistical weight of the $Y(n S)$ data set in the average. The largest fluctuation is $\langle\mathcal{S}\rangle=3.3$. Our set of 1951 overlapping fit regions corresponds to $\approx 1500$ independent measurements [29]. We determine the probability to observe a fluctuation of $\langle\mathcal{S}\rangle=3.3$ or larger in such a sample to be at least $45 \%$.

We do not observe any significant signal at $m_{A^{0}}=$ $0.214 \mathrm{GeV}$ (Fig. 5 in [21]) and set an upper limit on the coupling $f_{Y}^{2}\left(m_{A^{0}}=0.214 \mathrm{GeV}\right)<1.6 \times 10^{-6}$ at $90 \%$ C.L. (assuming $\mathcal{B}_{\mu \mu}=1$ ), which is significantly smaller than the value required to explain the HyperCP events as light Higgs production [11].

A fit to the $\eta_{b}$ region (Fig. 6 in [21]) includes background contributions from the ISR process $e^{+} e^{-} \rightarrow$ $\gamma_{\mathrm{ISR}} Y(1 S)$ and from the cascade decays $Y(n S) \rightarrow \gamma_{2} \chi_{b J}$, $\chi_{b J} \rightarrow \gamma_{1} Y(1 S)$ with $Y(1 S) \rightarrow \mu^{+} \mu^{-}$. We measure the rate of the ISR events in the $Y(4 S)$ data set, scale it to the $Y(2 S)$ and $Y(3 S)$ data, and fix this contribution in the fit. The rate of the cascade decays, the number of signal events, and the continuum background are free in the fits to

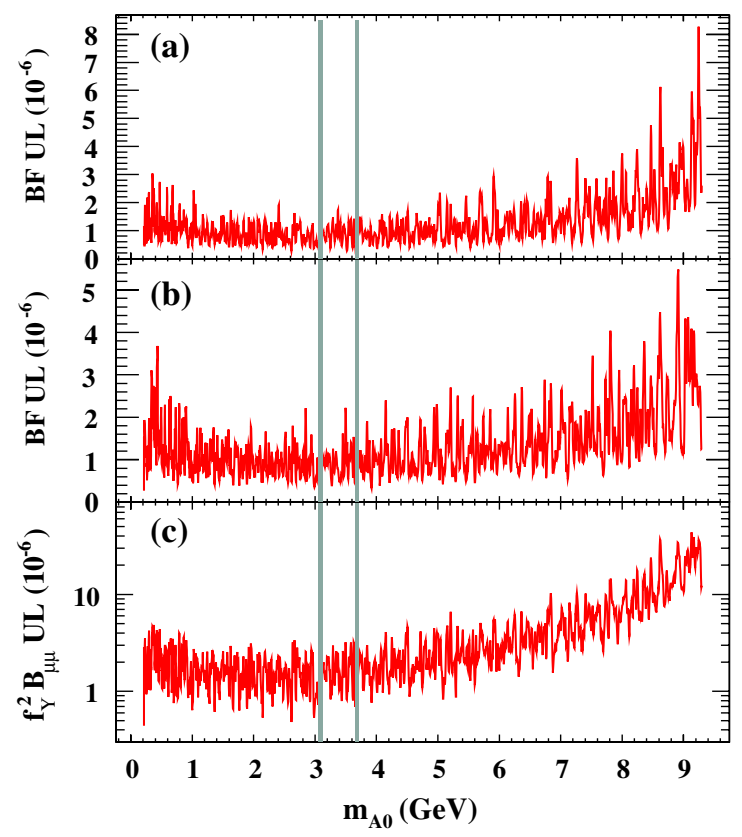

FIG. 2 (color online). $90 \%$ C.L. upper limits on (a) $\mathcal{B}\left(Y(2 S) \rightarrow \gamma A^{0}\right) \times \mathcal{B}_{\mu \mu}$, (b) $\mathcal{B}\left(Y(3 S) \rightarrow \gamma A^{0}\right) \times \mathcal{B}_{\mu \mu}$, and (c) effective coupling $f_{Y}^{2} \times \mathcal{B}_{\mu \mu}$ as a function of $m_{A^{0}}$. The shaded areas show the regions around the $J / \psi$ and $\psi(2 S)$ resonances excluded from the search. 
the $Y(2 S)$ and $Y(3 S)$ data sets. We measure $\mathcal{B}(Y(2 S) \rightarrow$ $\left.\gamma \eta_{b}\right) \times \mathcal{B}\left(\eta_{b} \rightarrow \mu^{+} \mu^{-}\right)=(-0.4 \pm 3.9 \pm 1.4) \times 10^{-6}$ and $\quad \mathcal{B}\left(Y(3 S) \rightarrow \gamma \eta_{b}\right) \times \mathcal{B}\left(\eta_{b} \rightarrow \mu^{+} \mu^{-}\right)=(-1.5 \pm$ $2.9 \pm 1.6) \times 10^{-6}$, where the first uncertainty is statistical and the second is systematic, dominated by the uncertainty in $\Gamma\left(\eta_{b}\right)$. Taking into account the BABAR measurements of $\mathcal{B}\left(Y(2 S) \rightarrow \gamma \eta_{b}\right)$ and $\mathcal{B}\left(Y(3 S) \rightarrow \gamma \eta_{b}\right)$ [17], we derive $\mathcal{B}\left(\eta_{b} \rightarrow \mu^{+} \mu^{-}\right)=(-0.25 \pm 0.51 \pm 0.33) \%$ and $\mathcal{B}\left(\eta_{b} \rightarrow \mu^{+} \mu^{-}\right)<0.9 \%$ at $90 \%$ C.L. This limit is consistent with the mesonic interpretation of the $\eta_{b}$ state.

In summary, we find no evidence for the dimuon decays of a light scalar particle in radiative decays of $Y(2 S)$ and $Y(3 S)$ mesons. We set upper limits on the coupling $f_{Y}^{2} \times$ $\mathcal{B}_{\mu \mu}$ for $0.212 \leq m_{A^{0}} \leq 9.3 \mathrm{GeV}$. Assuming $\mathcal{B}_{\mu \mu} \approx 1$ in the mass range $2 m_{\mu} \leq m_{A^{0}} \leq 1 \mathrm{GeV}$, our results limit the coupling $f_{Y}$ to be at most $12 \%$ of the standard model coupling of the $b$ quark to the Higgs boson. Our limits rule out much of the parameter space allowed by the light Higgs [9] and axion [6] models. We also set an upper limit on the dimuon branching fraction of the $\eta_{b}$.

We are grateful for the excellent luminosity and machine conditions provided by our PEP-II colleagues and for the substantial dedicated effort from the computing organizations that support BABAR. The collaborating institutions thank SLAC for its support and kind hospitality. This work is supported by DOE and NSF (USA), NSERC (Canada), CEA and CNRS-IN2P3 (France), BMBF and DFG (Germany), INFN (Italy), FOM (The Netherlands), NFR (Norway), MES (Russia), MEC (Spain), and STFC (United Kingdom). Individuals have received support from the Marie Curie EIF (European Union) and the A.P. Sloan Foundation.

*Deceased.

${ }^{\dagger}$ Now at Temple University, Philadelphia, PA 19122, USA.

*Also with Università di Perugia, Dipartimento di Fisica, Perugia, Italy.

${ }^{\S}$ Also with Università di Roma La Sapienza, I-00185 Roma, Italy.

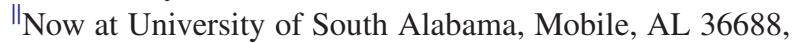
USA.

${ }^{\text {II }}$ Also with Laboratoire de Physique Nucléaire et de Hautes Energies, IN2P3/CNRS, Université Pierre et Marie CurieParis6, Université Denis Diderot-Paris7, F-75252 Paris, France.

**Also with Università di Sassari, Sassari, Italy.

[1] P. W. Higgs, Phys. Rev. Lett. 13, 508 (1964).

[2] S. Weinberg, Phys. Rev. Lett. 19, 1264 (1967); A. Salam, in Elementary Particle Theory, edited by N. Svartholm (Almquist and Wiksells, Stockholm, 1969), p. 367; S. L.
Glashow, J. Iliopoulos, and L. Maiani, Phys. Rev. D 2, 1285 (1970).

[3] R. Barate et al. (LEP Working Group for Higgs boson searches), Phys. Lett. B 565, 61 (2003).

[4] LEP-SLC Electroweak Working Group, Phys. Rep. 427, 257 (2006).

[5] R. Dermisek and J. F. Gunion, Phys. Rev. Lett. 95, 041801 (2005).

[6] Y. Nomura and J. Thaler, Phys. Rev. D 79, 075008 (2009).

[7] R. Dermisek and J. F. Gunion, Phys. Rev. D 73, 111701(R) (2006).

[8] F. Wilczek, Phys. Rev. Lett. 39, 1304 (1977).

[9] R. Dermisek, J. F. Gunion, and B. McElrath, Phys. Rev. D 76, 051105(R) (2007).

[10] H. Park et al. (HyperCP Collaboration), Phys. Rev. Lett. 94, 021801 (2005).

[11] X. G. He, J. Tandean, and G. Valencia, Phys. Rev. Lett. 98, 081802 (2007).

[12] M. L. Mangano and P. Nason, Mod. Phys. Lett. A 22, 1373 (2007).

[13] B. Aubert et al. (BABAR Collaboration), arXiv:0808.0017.

[14] W. Love et al. (CLEO Collaboration), Phys. Rev. Lett. 101, 151802 (2008).

[15] B. Aubert et al. (BABAR Collaboration), arXiv:0906.2219 [Phys. Rev. Lett. (to be published)].

[16] E. Fullana and M. A. Sanchis-Lozano, Phys. Lett. B 653, 67 (2007).

[17] B. Aubert et al. (BABAR Collaboration), Phys. Rev. Lett. 101, 071801 (2008); arXiv:0903.1124 [Phys. Rev. Lett. (to be published)].

[18] B. Aubert et al. (BABAR Collaboration), Nucl. Instrum. Methods Phys. Res., Sect. A 479, 1 (2002).

[19] M. Andreotti et al. (BABAR LST Collaboration), SLAC Report No. SLAC-PUB-12205.

[20] This corresponds to $\tau\left(A^{0}\right)<0.3 \mathrm{ps}$ for $m_{A^{0}}=0.212 \mathrm{GeV}$.

[21] See EPAPS Document No. E-PRLTAO-103-025935 for additional plots. For more information on EPAPS, see http://www.aip.org/pubservs/epaps.html.

[22] The BABAR detector Monte Carlo simulation is based on: S. Agostinelli et al. (GEANT4 Collaboration) Nucl. Instrum. Methods Phys. Res., Sect. A 506, 250 (2003).

[23] D. Lange, Nucl. Instrum. Methods Phys. Res., Sect. A 462, 152 (2001)

[24] M. J. Oreglia, Ph.D. thesis, Stanford University (Report No. SLAC-236, 1980, Appendix D); J.E. Gaiser, Ph.D. thesis, Stanford University (Report No. SLAC-255, 1982, Appendix F); T. Skwarnicki, Ph.D. thesis, Krakow INP (DESY Report No. F31-86-02, 1986, Appendix E).

[25] S. Jadach, B.F. Ward, and Z. Was, Comput. Phys. Commun. 130, 260 (2000).

[26] P. Nason, Phys. Lett. B 175, 223 (1986).

[27] R Barbieri et al., Phys. Lett. 57B, 455 (1975).

[28] M. Beneke, A. Signer, and V. A. Smirnov, Phys. Rev. Lett. 80, 2535 (1998).

[29] B. Aubert et al. (BABAR Collaboration), arXiv:0902.2176. 